\title{
Solution of an inverse adsorption problem with an epidemic genetic algorithm and the generalized extremal optimization algorithm
}

Ana Paula C. Cuco , Antônio J. Silva Neto , Haroldo F. Campos Velho \& Fabiano L. de Sousa

To cite this article: Ana Paula C. Cuco, Antônio J. Silva Neto, Haroldo F. Campos Velho \& Fabiano L. de Sousa (2009) Solution of an inverse adsorption problem with an epidemic genetic algorithm and the generalized extremal optimization algorithm, Inverse Problems in Science and Engineering; Formerly Inverse Problems in Engineering, 17:3, 289-302, DOI: 10.1080/17415970802083201

To link to this article: https://doi.org/10.1080/17415970802083201

\section{Published online: 24 Mar 2009.}

\section{Submit your article to this journal $\llbracket$}

Џ Article views: 47

Citing articles: 6 View citing articles $\square$ 


\title{
Solution of an inverse adsorption problem with an epidemic genetic algorithm and the generalized extremal optimization algorithm
}

\author{
Ana Paula C. Cuco a , Antônio J. Silva Neto ${ }^{a}$, \\ Haroldo F. Campos Velho ${ }^{\text {b* }}$ and Fabiano L. de Sousa ${ }^{c}$ \\ ${ }^{a}$ Department of Mechanical Engineering and Energy, Instituto Politécnico, IPRJ, Universidade \\ do Estado do Rio de Janeiro (UERJ), Nova Friburgo, RJ, Brazil; ${ }^{b}$ Laboratório Associado de \\ Computacão e Matemática Aplicada, Instituto Nacional de Pesquisas Espaciais (INPE), \\ São José dos Campos, SP, Brazil; ; Divisão de Mecânica Espacial e Controle, Instituto Nacional \\ de Pesquisas Espaciais (INPE), São José dos Campos, SP, Brazil
}

(Received 16 April 2007; final version received 30 November 2007)

\begin{abstract}
In the present work two recently developed stochastic methods, the epidemic genetic algorithm and the generalized extremal optimization algorithm are used for the solution of an inverse mass transfer problem, which is implicitly formulated as an optimization problem, for the estimation of parameters associated with the adsorption of biomolecules in resin beds. The estimates obtained with both methods present good accuracy, even in the presence of noisy data, provided that the model and experiment used are sensitive to the parameters being estimated. With Thomas' model for the direct mass transfer problem and real experimental data for lisozyme in adsorption columns, it is possible to estimate the maximum adsorption capacity in Langmuir's adsorption isotherm.
\end{abstract}

Keywords: adsorption; optimization; Thomas' model; inverse problems

\section{Introduction}

Due to the relevant applications in the food and pharmaceutical industries, there is a growing demand for the formulation and solution of direct and inverse mass transfer problems [1-5]. A high demand for purification processes for increasingly complex substances has been observed in recent years, with one of the most promising alternatives being the simulated moving bed (SMB) chromatography. For a full understanding of the operation and optimization [6] of SMBs, and a possible scale-up to industrial production, an accurate knowledge of mass transfer mechanisms and their dependence on the physicochemical and process parameters involved is required. The first step in that direction consists of the characterization of adsorption columns [7].

Kowalczyk et al. [8] estimated the pore-size distribution function that is used as a quantitative characteristic of the porous structure of solid adsorbents with respect to their heterogeneity, and Kowalczyk et al. [9] used a simple adsorption genetic algorithm (GA) for the estimation of parameters in a equation that models the adsorption process in a homogeneous micropore system.

*Corresponding author. Email: haroldo@lac.inpe.br 
The estimation of adsorption parameters for chromatography systems is a very important step in the column characterization used in the design of continuous separation processes based on the adsorption phenomenon, such as in simulated moving beds. For that purpose Forssén et al. [10] and Câmara et al. [11] used the least squares approach, the former having calculated the Jacobian matrix with numerical differentiation with complex variables. Vasconcellos et al. [12] used GAs. Lage et al. [13] have also used GA and a hybridization of GA with the Levenberg-Marquardt method (GA-LM), while Lage et al. [14] applied artificial neural networks (ANNs) developed for the solution of the inverse mass transfer problem, and Lage et al. [15] used a hybridization of ANNs with the Levenberg-Marquardt method (LM).

Yu et al. [16] and Ziyang et al. [17] have estimated adsorption equilibrium constants and kinetic parameters using GA. James et al. [18] estimated adsorption isotherms coefficients with the conjugate gradient method, and Zhang et al. [19] used a variation of GA for the same purpose.

In the present work, two stochastic methods are applied for the estimation of adsorption isotherm coefficients: (i) the generalized extremal optimization (GEO) [20]; and (ii) the epidemic genetic algorithm (EGA) [21].

For the solution of the direct problem Thomas' model is used, which provides accurate results when the effects of axial dispersion are negligible in comparison with other mass transfer mechanisms [7,22,23].

\section{Mathematical formulation and solution of the direct problem}

A mobile liquid phase composed by a diluted solution of the adsorbate of interest, for example a biomolecule, percolates through a resin bed, the solid fixed phase. The adsorbate is transferred from the bulk of the solution to the vicinity of the resin particles, i.e. a mass transfer mechanism through the liquid film, and then it diffuses to the interior of the particle pores being then adsorbed by the solid matrix.

The mathematical model for the separation chromatographic process is based on the mass balance for the two phases; one for the mobile phase that flows through the macroscale porous fixed resin bed, and the other for the resin particles involving the microscale porous solid matrix [24].

For the particular case in which the axial dispersion may be neglected, and the adsorbate inflow to the column of resins is constant, with concentration $C_{0}$, the adsorption problem described here has an analytical solution [25-27], which was first derived by Thomas [28]. According to this solution, the adsorbate concentration in the mobile liquid phase at the exit of the adsorption column, $C$, as a function of time, $t$, i.e. the breakthrough curve, is given by

$$
\frac{C}{C_{0}}=\frac{J(\eta / \sigma, \eta T)}{J(\eta / \sigma, \eta T)+\left\{[1-J(\eta / \sigma, \eta T)] \exp \left[\left(1-\sigma^{-1}\right)(\eta-\eta T)\right]\right\}}
$$

where

$$
\begin{gathered}
\sigma=1+C_{0} / k_{\mathrm{d}} \\
\eta=q_{\mathrm{m}} k_{1} h A_{\mathrm{c}} / Q \\
T=Q t\left(k_{\mathrm{d}}+C_{0}\right) / A_{\mathrm{c}} q_{\mathrm{m}} h
\end{gathered}
$$


$Q$ is the volumetric flow rate, $h$ is the total length of the adsorption column, $A_{\mathrm{c}}$ is the cross section of the column, $k_{\mathrm{d}}$ is the dissociation rate constant defined as

$$
k_{\mathrm{d}}=\frac{k_{2}}{k_{1}}
$$

$k_{1}$ is the adsorption rate constant, $k_{2}$ is the desorption rate constant and $q_{\mathrm{m}}$ is the maximum adsorbate concentration that the adsorbent may adsorb (maximum adsorption capacity), according to the Langmuir adsorption isotherm,

$$
q=\frac{q_{\mathrm{m}} C}{k_{\mathrm{d}}+C} .
$$

The function $J$ in Equation (1) corresponds to

$$
J(a, b)=1-e^{-b} \int_{0}^{a} e^{-\xi} I_{0}(2 \sqrt{b \xi}) \mathrm{d} \xi
$$

where $I_{0}$ is the modified Bessel function of the first kind and order zero, which may be approximated by an asymptotic series whose two first terms are given by

$$
J(a, b) \approx \frac{1}{2}[1-\operatorname{erf}(\sqrt{a}-\sqrt{b})]+\frac{\exp \left[1-(\sqrt{a}-\sqrt{b})^{2}\right]}{2 \pi^{1 / 2}\left[(a b)^{1 / 4}+b^{1 / 2}\right]} .
$$

When the geometry, initial condition, boundary conditions, physico-chemical and process parameters are known, we are able to calculate the concentration of the adsorbate at the exit of the column, i.e. $C(h, t)$. This is the direct problem, i.e. the determination of the breakthrough curve.

\section{Mathematical formulation and solution of the inverse problem}

Here, we are interested in the estimation of the coefficients in the adsorption isotherm given by Equation (6) using measured values of the adsorbate concentration at the exit of the column, i.e. $C_{\text {meas }_{i}}=C_{\text {meas }}\left(t_{i}\right)$ with $i=1,2, \ldots, N_{\mathrm{d}}$, where $N_{\mathrm{d}}$ represents the total number of experimental data available.

According to the sensitivity analysis performed by Folly et al. [7] we may try to estimate the vector of unknowns

$$
\overrightarrow{Z_{1}}=\left\{k_{\mathrm{d}}, q_{\mathrm{m}}\right\}
$$

but difficulties may arise due to low values of the sensitivity coefficients related to the parameter $k_{\mathrm{d}}$, and that behaviour was in fact observed by Lage et al. [15].

In the present work besides the estimation of $\vec{Z}_{1}$ we will also look into the estimation of

$$
\overrightarrow{Z_{2}}=\left\{q_{\mathrm{m}}\right\}
$$

fixing the value of $k_{\mathrm{d}}$, and also into the estimation of

$$
\overrightarrow{Z_{3}}=\left\{k_{\mathrm{d}}\right\}
$$

fixing the value of $q_{\mathrm{m}}$. 
As the number of experimental data available, $N_{\mathrm{d}}$, will be much larger than the number of unknowns considered, $N_{\mathrm{u}}=1$ or 2 , we formulate the inverse problem implicitly as an optimization problem in which we seek to minimize the cost function given by the summation of the squared residues between the calculated and measured adsorbate concentrations at the exit of the adsorption column, i.e. $C_{\text {calc }_{i}}$ and $C_{\text {meas }_{i}}$, respectively, with $i=1,2, \ldots, N_{\mathrm{d}}$,

$$
S(\vec{Z})=\sum_{i=1}^{N_{\mathrm{d}}}\left[C_{\mathrm{calc}_{i}}(\vec{Z})-C_{\text {meas }_{i}}\right]^{2} .
$$

In order to find the value of $\vec{Z}^{*}$ for which $S$ is minimum, we have used two recently proposed stochastic methods: (i) GEO [20]; and (ii) EGA [21]. Both the methods will be described next.

\section{The generalized extremal optimization algorithm}

GEO has been proposed and implemented recently [20], and was devised to be applied in complex optimization problems. Based on the Back-Sneppen simplified model of evolution [29], it has been applied successfully to design optimization problems [30,31] as well as to an inverse radiative transfer problem [32].

GEO makes no use of derivatives requiring only the solution of the direct problem given by Equations (1)-(4). The main steps of the canonical GEO algorithm are shown in Figure 1.

A very attractive feature of GEO is that it has only one free parameter to adjust, $\tau$, which may be considered an advantage when compared to other stochastic methods that have a larger number of parameters to be set.

In GEO, a string of $L$ bits, which encodes the $N_{\mathrm{u}}$ unknowns, is considered a population of species, i.e. each bit represents a species. Each bit is associated to a fitness number that is proportional to the gain, or loss, the cost function value has in flipping that particular bit. All bits are then ranked from $k=1$, for the least adapted bit, to $k=L$ for the best adapted. A bit is then mutated according to the probability distribution $P_{k} \propto k^{-\tau}$, where $k$ is the rank of a selected bit candidate to mutate. Making $\tau \rightarrow 0$ all bits have the same probability to mutate, whereas for $\tau \rightarrow \infty$ only the worst adapted bit will mutate.

In practice, it has been observed that the best value of $\tau$, i.e. the one that yields the best performance of the algorithm for a given application, usually lies in the range $(0.75,3.0)$. A detailed description of GEO can be found in Sousa et al. [20].

\section{The epidemic genetic algorithm}

Simple GAs operate on a fixed-sized population of fixed-length individuals, and in general the individuals are represented by a binary string that encodes the variables of the problem that the algorithm is trying to minimize.

In the present work, each individual is composed by the unknowns of the inverse problem, which are given by Equations (9)-(11), and in fact is represented by a real-valued string.

Simple GAs use basically three operators: selection, crossover and mutation. The selection operator identifies the fittest individuals of a given population to serve as parents 


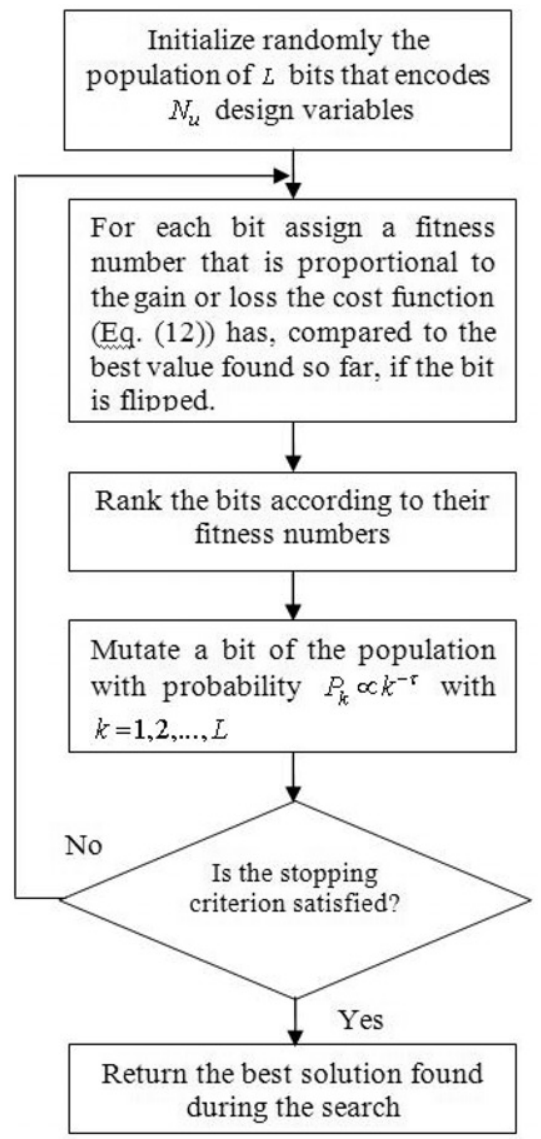

Figure 1. The canonical GEO algorithm.

of the next generation. The fitness value of each individual is related to the value of the cost function given by Equation (12). The selection operator ensures that the best fit individuals have a higher probability to be selected to reproduce and form a new generation.

The crossover operator randomly chooses a pair of individuals among those previously selected to breed and exchanges information between them.

The mutation operator is usually considered a secondary operator. Its main function is to restore diversity that may be lost from the repeated application of the selection and crossover operators. This operator simply takes one string from the population and randomly alters some value within it. Following the example of nature, the probability of applying the mutation operator is very low compared to the probability of applying the crossover operator.

In the present work, a fourth operator is applied, the epidemical strategy [21], leading to EGA. This operator is activated when a prescribed number of generations are reached without improvement of the best individual. Then, all the population is affected by a plague, and only those that have the best fit, say the top $10 \%$ individuals better fitted, survive. The remaining individuals die and are replaced by new individuals with new genetic variability, such as immigrants arriving in order to evolve the population. 
Two parameters need to be chosen: one determines when the strategy will be activated, i.e. the number of generations without improvement of the fitness value of the best individual, $N_{e}$, and the other determines the fraction of the population that will survive the plague, $f_{e}$.

Slow convergence is a particular problem for the stochastic optimization schemes, the same observation is valid to the GA. Alternative GA techniques have been designed to improve the convergence performance. Another challenge is to design a GA for dealing with multimodal function optimization. For attending to such features some genetic operators were proposed such as: micro-GA [33] and niching [34]. A niche is a particular sub-domain of the entire search space, where each niche has different nuances on each other. This is a natural feature to maintain the genotype diversity in a population [35-37], under insulation these niches are a mechanism for the speciation. Goldberg and Richardson [34] have introduced the method of sharing functions - now the method is known as sharing, and it is directly applicable to the multimodal function optimization [36]. Sharing is a natural algorithm to implement niching [38].

Another procedure to preserve the diversity in the GA population is the re-initialization. Mahfoud [36] has reported that Goldberg [39] has investigated such ideas, but re-initialization is not treated as a new GA operator. Micro-genetic algorithms are GAs with small populations with re-initialization. Krishnakumar's [33] micro-GA approach uses a population with five individuals, driven by elitism. He compared his micro-GA with standard GA (SGA), resulting in a faster and better solution to a engineering control problem. Other applications of micro-GA have been presented by Liu and Han [37]. We pointed out that Chakraborti et al. [40] addressed a solution, an important and difficult real-world problem, where it was shown to be of little additional advantage over the simple GA, when the objective function is stationary. Micro-GA deals with selection and crossover GA operators, while mutation is usually omitted [36,37]. On the other hand, EGA employs all standard GA operators, and also the typical population size of the SGA, but it introduces the epidemic strategy.

An interesting issue would be a comparison among the diversity preservation strategies (niching, micro-GA, EGA, for example). This will be the focus of a future work. Here, the goal is just the performance comparison between GEO and EGA.

\section{Results and discussion}

Because of the availability of real experimental data, we have chosen the system investigated by Chase [26] for the substance lisozyme as our test case. Table 1 presents the process and physico-chemical parameters used.

Table 1. Process and physico-chemical parameters for Chase's experiment with lisozyme [26].

\begin{tabular}{lc}
\hline Parameter & Value \\
\hline$H-$ column height $(\mathrm{cm})$ & 10.4 \\
$A_{\mathrm{c}}-$ column cross-section $\left(\mathrm{cm}^{2}\right)$ & 0.785 \\
$Q-$ volumetric flow rate $\left(\mathrm{mL} \mathrm{min}^{-1}\right)$ & 1.0 \\
$C_{0}-$ adsorbate concentration at the column inlet $\left(\mathrm{mg} \mathrm{mL}^{-1}\right)$ & 0.1 \\
$q_{\mathrm{m}}-$ maximum adsorption capacity $\left(\mathrm{mg} \mathrm{mL}^{-1}\right)$ & 14 \\
$k_{\mathrm{d}}-$ dissociation rate constant $\left(\mathrm{mg} \mathrm{mL} \mathrm{mL}^{-1}\right)$ & 0.025 \\
$k_{1}-$ adsorption rate constant $\left(\mathrm{mL} \mathrm{mg} \cdot \mathrm{min}^{-1}\right)$ & 0.20 \\
\hline
\end{tabular}


The values of $k_{1}, k_{\mathrm{d}}$ and $q_{\mathrm{m}}$ shown in Table 1 were obtained using a batch experiment and considering a Langmuir adsorption isotherm.

From the sensitivity analysis performed by Folly et al. [7] one concludes that using Thomas' model and the experimental data obtained by Chase [26] for lisozyme it is not possible to estimate $k_{1}$. Therefore, in all computations, the results of which will be presented next, a fixed value for this parameter has been considered, i.e. the one shown in Table 1.

Tables 2 and 3 show the estimates obtained for the vector of unknowns $\vec{Z}_{1}$, see Equation (9), for lisozyme, using GEO and EGA, respectively, and Chase's experimental

Table 2. Estimates for $k_{\mathrm{d}}$ and $q_{\mathrm{m}}$ for lisozyme using GEO with $\tau=1.25$ and Chase's experimental data [26]. $d=0.11514$ (Equation (13)).

\begin{tabular}{lccc}
\hline Run & $k_{\mathrm{d}}\left(\mathrm{mg} \mathrm{mL}^{-1}\right)$ & $q_{\mathrm{m}}\left(\mathrm{mg} \mathrm{mL}^{-1}\right)$ & $\begin{array}{c}S \text { Equation }(12) \\
\left(\mathrm{mg} \mathrm{mL}^{-1}\right)^{2}\end{array}$ \\
\hline 1 & 0.0272 & 11.2862 & 0.0251 \\
2 & 0.0335 & 11.8862 & 0.0243 \\
3 & 0.0350 & 14.3182 & 0.0233 \\
4 & 0.0287 & 14.0856 & 0.0327 \\
5 & 0.0258 & 13.2334 & 0.0278 \\
6 & 0.0325 & 12.0309 & 0.0231 \\
7 & 0.0321 & 12.7347 & 0.0254 \\
8 & 0.0215 & 12.7701 & 0.0236 \\
9 & 0.0325 & 13.9952 & 0.0234 \\
10 & 0.0316 & 11.0701 & \\
Average $\mu$ & 0.0300 & 12.7411 & \\
Standard deviation $\sigma$ & 0.0042 & 1.1669 & \\
$\sigma$ & 13.82 & 9.16 & \\
\hline$\mu 100 \%$ & & & \\
\hline
\end{tabular}

Table 3. Estimates for $k_{\mathrm{d}}$ and $q_{\mathrm{m}}$ for lisozyme using EGA with an initial population of 100 individuals, Chase's experimental data [26] and $n_{e}=3$ and $f_{e}=0.1 . d=0.047396$ (Equation (13)).

\begin{tabular}{lccc}
\hline Run & $k_{\mathrm{d}}\left(\mathrm{mg} \mathrm{mL}^{-1}\right)$ & $q_{\mathrm{m}}\left(\mathrm{mg} \mathrm{mL}^{-1}\right)$ & $\begin{array}{c}S \text { Equation }(12) \\
\left(\mathrm{mg} \mathrm{mL}^{-1}\right)^{2}\end{array}$ \\
\hline 1 & 0.0372 & 14.0389 & 0.0240 \\
2 & 0.0455 & 14.1111 & 0.0369 \\
3 & 0.0475 & 14.2639 & 0.0270 \\
4 & 0.0448 & 14.1780 & 0.0210 \\
5 & 0.0446 & 14.1892 & 0.0205 \\
6 & 0.0444 & 14.1681 & 0.0205 \\
7 & 0.0406 & 14.0509 & 0.0208 \\
8 & 0.0397 & 14.0909 & 0.0221 \\
9 & 0.0374 & 13.9978 & 0.0241 \\
10 & 0.0395 & 14.0770 & 0.0215 \\
Average $\mu$ & 0.0421 & 14.1166 & \\
Standard deviation $\sigma$ & 0.0037 & 0.0817 & \\
$\sigma$ & 8.74 & 0.58 & \\
\hline
\end{tabular}


data [26]. Each table shows the results obtained for 10 different runs of each algorithm, as well as the average and the standard deviation for each unknown. It also shows the value of a measure of the dispersion of the estimates given by

$$
d=\sqrt{\frac{\left(1+\left(\sigma_{q_{\mathrm{m}}} / \bar{q}_{\mathrm{m}}\right)\right)^{2}+\left(1+\left(\sigma_{k_{\mathrm{d}}} / \bar{k}_{\mathrm{d}}\right)\right)^{2}}{2}}-1 .
$$

Figures 2 and 3 show graphically the dispersion of the results obtained with the stochastic methods GEO and EGA, respectively. Observe that the scales used in the two figures are different.

Figure 4 shows Chase's experimental data [26] and the breakthrough curves calculated using the average values obtained for $q_{\mathrm{m}}$ and $k_{\mathrm{d}}$, shown in Tables 2 and 3, using GEO and EGA, respectively.

From the results presented we conclude that the low sensitivity to the parameter $k_{\mathrm{d}}$ may be affecting negatively the estimation of $q_{\mathrm{m}}$ when the two parameters are estimated simultaneously.

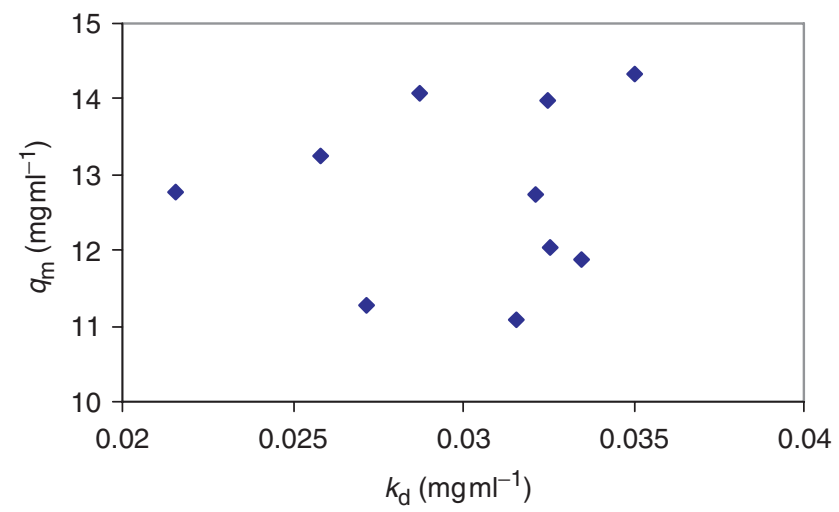

Figure 2. Dispersion of the results obtained with GEO.

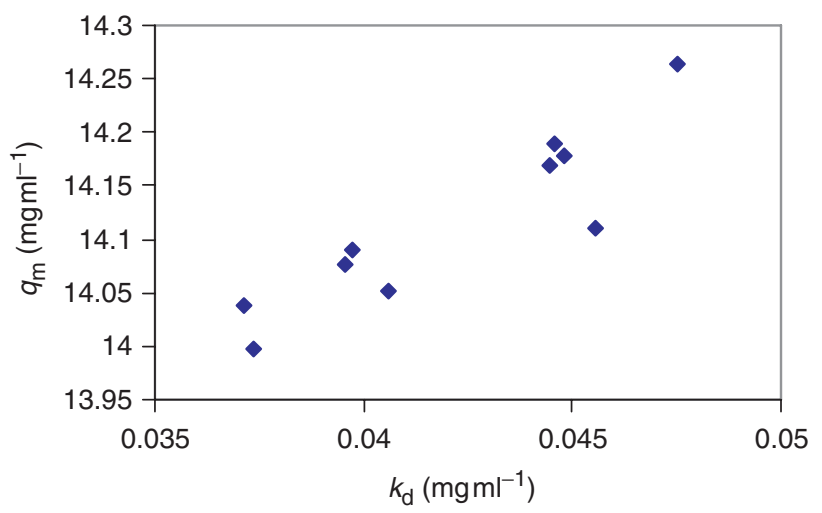

Figure 3. Dispersion of the results obtained with EGA. 
We may also conclude that with the control parameters chosen for GEO and EGA the latter presents a lower dispersion of the estimates. This fact is confirmed by the value of the measure $d$, given by Equation (13), shown in Tables 2 and 3 .

We have then fixed the value of $q_{\mathrm{m}}$ as $14 \mathrm{mg} \mathrm{mL}^{-1}$ and have estimated $k_{\mathrm{d}}$ using both GEO and EGA. The results obtained after 10 runs of both algorithms are shown in Table 4.

Even though the dispersion of the estimates is smaller with EGA we observe from Tables 1 and 4 that the estimated average value for $k_{\mathrm{d}}$ obtained with GEO is closer to the experimental value shown in Table 1 . Nonetheless, as mentioned before, due to the low value of the sensitivity coefficient related to this parameter the estimates may not be accurate.

Figure 5 shows Chase's experimental data and the breakthrough curves calculated using the values for $q_{\mathrm{m}}$ and $k_{\mathrm{d}}$ shown in Table 4 .

Finally, we have varied the value of $k_{\mathrm{d}}$ in the range from $0.015 \mathrm{mg} \mathrm{mL}^{-1}$ up to $0.035 \mathrm{mg} \mathrm{mL}^{-1}$, and for each fixed value of $k_{\mathrm{d}}$ we have estimated $q_{\mathrm{m}}$ using both GEO and EGA. The results obtained after 10 runs of both algorithms are shown in Tables 5 and 6 .

Figures 6 and 7 show Chase's experimental data [26] and the calculated breakthrough curves for lisozyme with the fixed value of $k_{\mathrm{d}}$ and the estimated values of $q_{\mathrm{m}}$ with GEO and EGA, respectively.

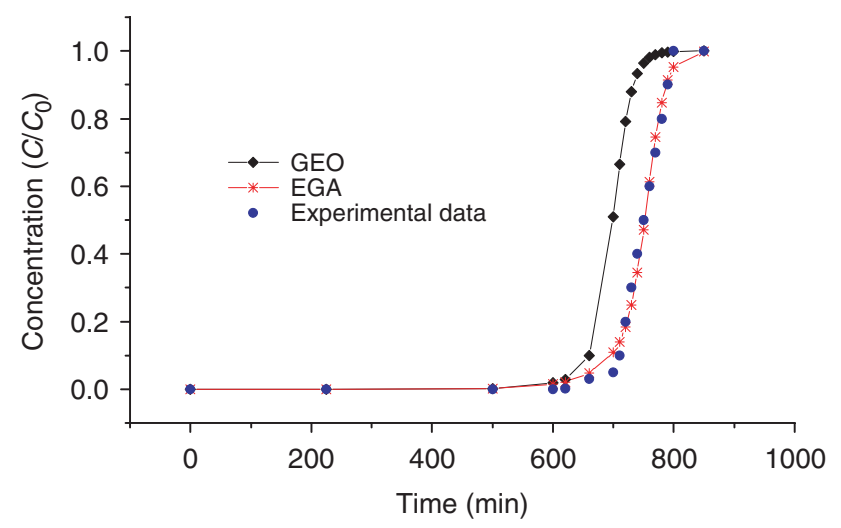

Figure 4. Chase's experimental data [26] and calculated breakthrough curves using the average of the estimated values for $q_{\mathrm{m}}$ and $k_{\mathrm{d}}$ with GEO and EGA (Tables 2 and 3 ).

Table 4. Estimated values for $k_{\mathrm{d}}$, being $q_{\mathrm{m}}$ fixed at $14 \mathrm{mg} \mathrm{mL}^{-1}$, after 10 runs of GEO and EGA.

\begin{tabular}{lll}
\hline & GEO & \multicolumn{1}{c}{ EGA } \\
\hline$\overline{k_{\mathrm{d}}}\left(\mathrm{mg} \mathrm{mL}^{-1}\right)$ & 0.0261 & 0.0381 \\
$\sigma_{k_{\mathrm{d}}}\left(\mathrm{mg} \mathrm{mL}^{-1}\right)$ & 0.0009 & $4.438 \times 10^{-5}$ \\
$\overline{\sigma_{k_{\mathrm{d}}}} \times 100 \%$ & 3.34 & 0.12 \\
$\overline{\overline{k_{\mathrm{d}}}}$ & & \\
\hline
\end{tabular}




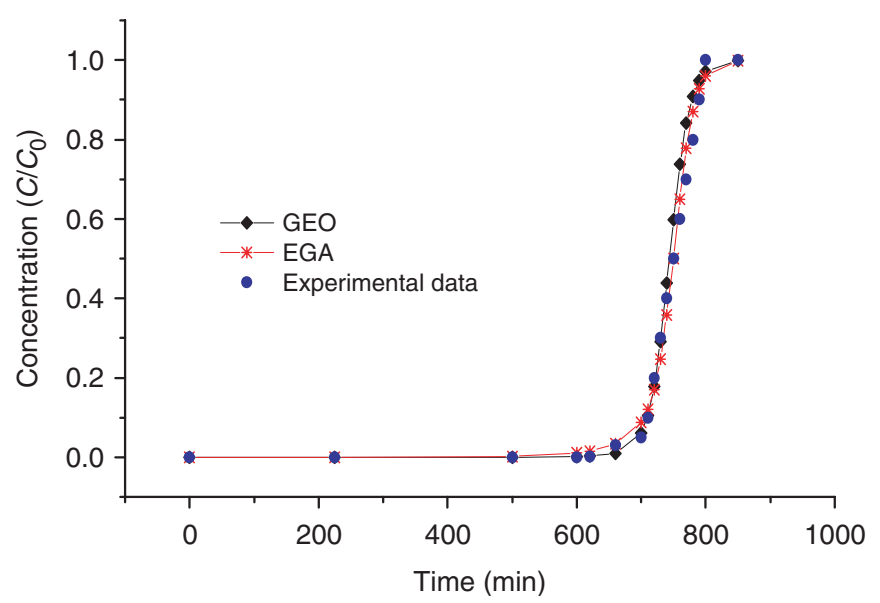

Figure 5. Chase's experimental data [26] and calculated breakthrough curves using the average estimated values for $k_{\mathrm{d}}$ with GEO and EGA being $q_{\mathrm{m}}$ fixed at $14 \mathrm{mg} \mathrm{mL}^{-1}$ (Table 4).

Table 5. Estimated values for $q_{\mathrm{m}}$, being $k_{\mathrm{d}}$ fixed, after 10 runs of GEO.

\begin{tabular}{lccc}
\hline $\mathrm{k}_{\mathrm{d}}\left(\mathrm{mg} \mathrm{mL}^{-1}\right)$ & $\bar{q}_{\mathrm{m}}\left(\mathrm{mg} \mathrm{mL}^{-1}\right)$ & $\sigma_{q_{\mathrm{m}}}\left(\mathrm{mg} \mathrm{mL}^{-1}\right)$ & $\frac{\sigma_{q_{\mathrm{m}}}}{\bar{q}_{\mathrm{m}}} \times 100 \%$ \\
\hline 0.015 & 14.92 & 0.148 & 0.99 \\
0.020 & 14.52 & 0.137 & 0.94 \\
0.025 & 14.10 & 0.184 & 1.31 \\
0.030 & 14.01 & 0.153 & 1.09 \\
0.035 & 13.99 & 0.139 & 1.00 \\
\hline
\end{tabular}

Table 6. Estimated values for $q_{\mathrm{m}}$, being $k_{\mathrm{d}}$ fixed, after 10 runs of EGA.

\begin{tabular}{llll}
\hline $\mathrm{k}_{\mathrm{d}}\left(\mathrm{mg} \mathrm{mL}^{-1}\right)$ & $\bar{q}_{m}\left(\mathrm{mg} \mathrm{mL}^{-1}\right)$ & $\sigma_{q_{\mathrm{m}}}\left(\mathrm{mg} \mathrm{mL}^{-1}\right)$ & $\frac{\sigma_{q_{\mathrm{m}}}}{\bar{q}_{\mathrm{m}}} \times 100 \%$ \\
\hline 0.015 & 15.0 & 0 & 0 \\
0.020 & 14.507 & 0.0035 & 0.02 \\
0.025 & 14.201 & 0.0014 & 0.01 \\
0.030 & 14.046 & 0.0006 & 0.005 \\
0.035 & 14.007 & 0.0004 & 0.003 \\
\hline
\end{tabular}

From the results shown in Tables 5 and 6 we observe a good agreement with the experimental value of $q_{\mathrm{m}}$ shown in Table 1, and again it seems that the dispersion of the estimates is smaller with EGA.

From Figures 6 and 7 it is observed that $k_{\mathrm{d}}$, as expected, seems to have a negligible effect on the breakthrough curves.

All results shown for GEO were obtained using 5000 evaluations of the cost function. The dispersion of the estimates may become smaller if a larger number of function 


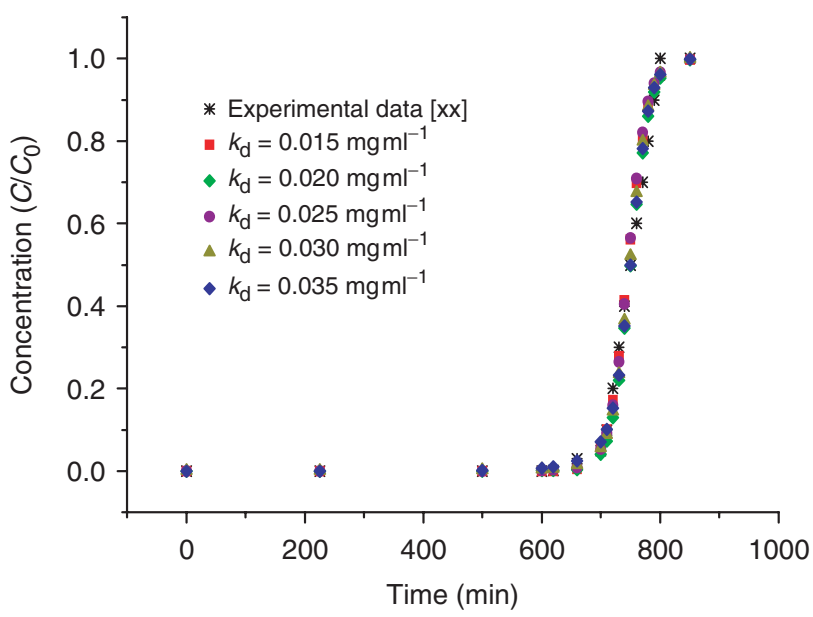

Figure 6. Chase's experimental data [26] and calculated breakthrough curves using the fixed value of $k_{\mathrm{d}}$ and the average estimated values for $q_{\mathrm{m}}$ with GEO (Table 5).

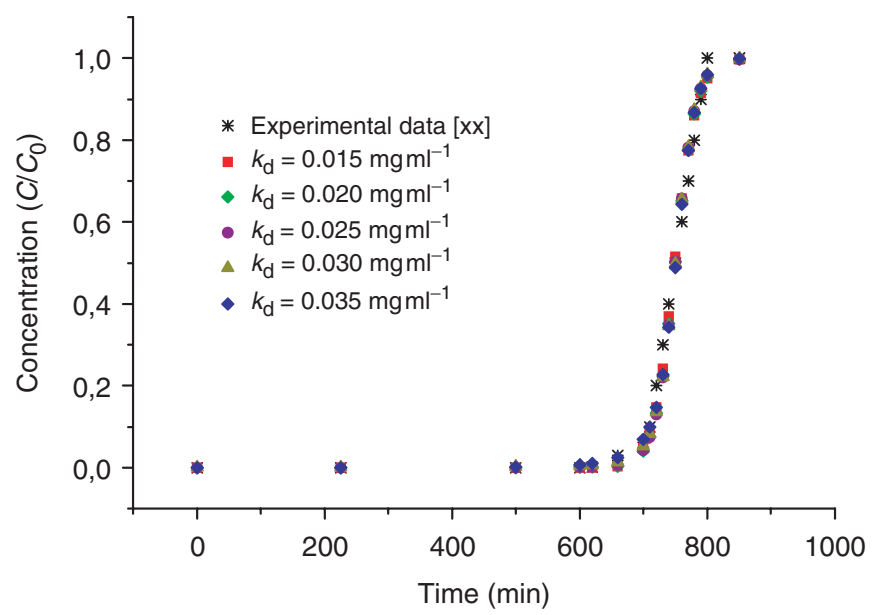

Figure 7. Chase's experimental data [26] and calculated breakthrough curves using the fixed value of $k_{\mathrm{d}}$ and the average estimated values for $q_{\mathrm{m}}$ with EGA (Table 6).

evaluations is considered. Of course a higher amount of CPU time will be required. This subject must be further investigated.

Due to the simplicity of the solution obtained for the direct problem with Thomas' model, only 10 generations were required to obtain the results presented before using EGA. Tests were performed with a total of 100 or 1000 generations, nonetheless no improvement was observed in the value of the cost function.

Concerning the accuracy of the solutions found by GEO and EGA when compared to the experimental data, for the results with fixed $k_{\mathrm{d}}$, both algorithms presented similar average results, with the EGA ones presenting a lower dispersion. On the other hand, when estimating $k_{\mathrm{d}}$ and $q_{\mathrm{m}}$ simultaneously, the EGA had clearly a better performance than GEO. 


\section{Conclusions}

The application of two stochastic methods, EGA and GEO yielded good estimates for the maximum adsorption capacity, one parameter in Langmuir's adsorption isotherm, for a system with lisozyme in a diluted solution being adsorbed in resin beds.

It was observed that EGA tends to provide estimates with a smaller dispersion when compared to GEO. Nonetheless this conclusion cannot yet be generalized. A small number of function evaluations was considered for GEO. With a larger number of functions evaluations a reduction in the dispersion may occur.

The results obtained so far are very encouraging and the application of the recently developed stochastic methods in the solution of inverse mass transfer problems deserves further investigation.

\section{Acknowledgements}

The authors acknowledge the financial support provided by $\mathrm{CNPq}$, Conselho Nacional de Desnvolvimento Científico e Tecnológico, and FAPERJ, Fundação Carlos Chagas Filho de Amparo à Pesquisa do Estado do Rio de Janeiro.

\section{References}

[1] G. Guiochon, Preparative liquid chromatography (Review), J. Chromat. 965 (2002), pp. 129-161.

[2] F.R.C. Silva and C.C. Santana, Adsorption of inulinases in ion exchange columns, Appl. Biochem. Biotech. 84-86 (2000), pp. 1063-1078.

[3] A.M. Denisov, Inverse problems of absorption dynamics, Minisymposium on Inverse Problems in Medicine, Engineering and Geophysics, XXIII Brazilian Congress on Applied and Computational Mathematics, Santos, Brazil, 2000, pp. 24-32.

[4] J.F. Vasconcellos, A.J. Silva Neto, and C.C. Santana, An inverse mass transfer problem in solidliquid adsorption systems, Inv. Prob. Eng. 11 (2003), pp. 391-408.

[5] L.D.T. Câmara, C.C. Santana and A.J. Silva Neto, Kinetic modeling of properties adsorption with a methodology of error analysis. J. Separat. Sci. 30 (2007), pp. 688-692.

[6] G. Paredes and M. Mazzotti, Optimization of simulated moving bed and column chromatography for a plasmid DNA purification step and for a chiral separation, J. Chromat. A 1142 (2007), pp. 56-68.

[7] F.M. Folly, A.J. Silva Neto and C.C. Santana, An inverse mass transfer problem for the characterization of simulated moving beds adsorption columns, 5th International Conference on Inverse Problems in Engineering: Theory and Practice, Cambridge, UK, 2005.

[8] P. Kowalczyk, A.P. Terzyk, P.A. Gauden, R. Leboda, E. Szmechtig-Gauden, G. Rychlicki, Z. Ryu, and $\mathrm{H}$. Rong, Estimation of the pore-size distribution function from the nitrogen adsorption isotherm. Comparison of density functional theory and the method of Do and co-workers, Carbon 41 (2003), pp. 1113-1125.

[9] P. Kowalczyk, A.P. Terzyk, P.A. Gauden, and R. Leboda, The characterization of microporous activated carbons utilizing a Simple Adsorption Genetic Algorithm (SAGA) (Letter to the editor), J. Colloid Inter. Sci. 239 (2001), pp. 591-594.

[10] P. Forssén, R. Arnell, and T. Fornstedt, An improved algorithm for solving inverse problems in liquid chromatography, Comp. Chem. Eng. 30 (2006), pp. 1381-1391.

[11] L.D.T. Câmara, C.C. Santana and A.J. Silva Neto, Direct and inverse modeling of enzymes adsorption kinetics in macro-porous adsorbents, 14th Inverse Problems in Engineering Seminar, Ames, USA, 2006. 
[12] J.F.V. Vasconcellos, A.J. Silva Neto, C.C. Santanta and F.J.C.P. Soeiro, Parameter estimation in adsorption columns with a stochastic global optimization method, 4th International Conference on Inverse Problems in Engineering: Theory and Practice, II, pp. 227-234, Angra dos Reis, Brazil, 2002.

[13] L.F. Lage, A.P.C. Cuco, F.M. Folly, F.J.C.P. Soeiro and A.J. Silva Neto, Stochastic and hybrid methods for the solution of an inverse mass transfer problem, III European Conference on Computational Mechanics, Lisbon, Portugal 2006.

[14] L.F. Lage, A.P.C. Cuco, F.J.C.P. Soeiro and A.J. Silva Neto, Characterization of adsorption columns with Thomas' model and artificial neural networks, IV Brazilian National Congress of Mechanical Engineering, Recife, Brazil, 2006.

[15] L.F. Lage, A.P.C. Cuco, A.J. Silva Neto, F.M. Folly and F.J.C.P. Soeiro, Solution of an inverse mass transfer problem with a hybridization of artificial neural networks and LevenbergMarquardt method, 11th Brazilian Congress of Thermal Sciences and Engineering, Curitiba, Brazil, 2006.

[16] W. Yu, K. Hidajat, and A.K. Ray, Determination of adsorption and kinetic parameters for methyl acetate esterification and hydrolysis reaction catalyzed by Amberlyst 15, Appl. Cataly. A: Gen. 260 (2004), pp. 191-205.

[17] Z. Ziyang, K. Hidajat, and A.K. Ray, Determination of adsorption and kinetic parameters for methyl tert-butyl ether synthesis from tert-butyl alcohol and methanol, J. Cataly. 200 (2001), pp. 209-221.

[18] J. James, M. Sepúlveda, F. Charton, I. Quiñones, and G. Guiochon, Determination of binary competitive equilibrium isotherms from the individual chromatographic band profiles, Chem. Eng. Sci. 54 (1999), pp. 1677-1696.

[19] Y. Zhang, K. Hidajat, and A.K. Ray, Determination of competitive adsorption isotherm parameters of pindolol enantiometers on $\alpha_{1}$-acid glycoprotein chiral stationary phase, J. Chromat. A 1131 (2006), pp. 176-184.

[20] F.L. Sousa, F.M. Ramos, P. Paglione, and R.M. Girardi, New stochastic algorithm for design optimization, AIAA J. 41 (2003), pp. 1808-1818.

[21] L.D. Chiwiacowski and H.F. Campos Velho, Different approaches for the solution of a backward heat conduction problem, Inv. Prob. Eng. 11 (2003), pp. 471-494.

[22] A. Felinger, A. Cavazzini, and G. Guiochon, Numerical determination of the competitive isotherm of enantiometers, J. Chromat. A 986 (2003), pp. 207-225.

[23] W. Hao and J. Wang, Evaluation of nonlinear chromatographic performance by frontal analysis using a single multi-plate mathematical model, J. Chromat. A 1063 (2005), pp. $47-56$.

[24] G. Guiochon and B. Lin, Modeling for Preparative Chromatography, Academic Press, Great Britain, 2003.

[25] H.W. Blanch and D.S. Clark, Biochemical Engineering, Marcel Dekker, New York, 1997.

[26] H.A. Chase, Prediction of the performance of preparative affinity chromatography, J. Chromat. 297 (1984), pp. 179-202.

[27] G.R. Rice and D.D. Do, Applied Mathematics and Modeling for Chemical Engineers, John Wiley \& Sons, New York, 1994.

[28] H. Thomas, Heterogeneous ion exchange in flowing system, J. Am. Chem. Soc. 66 (1944), pp. 1664-1668.

[29] P. Bak and K. Sneppen, Punctuated equilibrium and criticality in a simple model of evolution, Phys. Rev. Lett. 71 (1993), pp. 4083-4086.

[30] V.V. Vlassov, F.L. de Sousa, and W.K. Takahashi, Comprehensive optimization of a heat pipe radiator assembly filled with ammonia or acetone, Int. J. Heat Mass Tran. 49 (2006), pp. 4584-4595.

[31] V.V. Vlassov, A.P.C. Cuco, F.L. de Sousa and A.J. Silva Neto, Design optimization of two-stage radiator with variable emittance: Analysis of concept feasibility, 11th Brazilian Congress of Thermal Sciences and Engineering, Curitiba, Brazil, 2006. 
[32] F.L. Sousa, F.J.C.P. Soeiro, A.J. Silva Neto, and F.M. Ramos, Application of the generalized extremal optimization algorithm to an inverse radiative transfer problem, Inv. Prob. Sci. Eng. 15 (2007), pp. 699-714.

[33] K. Krishnakumar, Micro-Genetic Algorithms for Stationary and Non-Stationary Function Optimization. Intell. Contr. Adap. Syst., SPIE 1196, SPIE, Bellingham, USA, 1989.

[34] D.E. Goldberg and J. Richardson, Genetic algorithms with sharing for multimodal function optimization, in Genetic Algorithms and Their Applications: Proceedings of the Second International Conference on Genetic Algorithms, J.J. Grefenstette, ed., Cambridge, USA, 1987, pp. 41-49.

[35] M.M. Melanie, An Introduction to Genetic Algorithms, MIT Press, Cambridge, USA, 1998.

[36] S.W. Mahfoud, Niching Methods for Genetic Algorithms, Computer Science Thesis, University of Illinois at Urbana-Champaign, USA, 1995.

[37] G.R. Liu and X. Han, Computational Inverse Techniques in Nondestructive Evaluation, CRC Press, Florida, USA, 2003.

[38] J. Horn, The Nature of Niching: Genetic Algorithms and the Evolution of Optimal, Cooperative Populations. Computer Science Thesis, University of Illinois at Urbana-Champaign, USA (1997).

[39] D.E. Goldberg, Sizing populations for serial and parallel genetic algorithms. Proceedings of the third International Conference on Genetic Algorithms, 1989, pp. 70-79.

[40] N. Chakraborti, K. Deb, and A. Jha, A genetic algorithm based heat transfer analysis of a bloom re-heating furnace, Steel Res. 71 (2000), pp. 396-402. 